\title{
Magnetic resonance imaging DTI-FT study on schizophrenic patients with typical negative first symptoms
}

\author{
CHENGYU GU, YING ZHANG, FUQUAN WEI, YOUGEN CHENG, YULIN CAO and HONGTAO HOU \\ Department of Geriatric, Tongde Hospital of Zhejiang Province, Hangzhou, Zhejiang 310012, P.R. China
}

Received March 23, 2016; Accepted June 17, 2016

DOI: $10.3892 /$ etm.2016.3469

\begin{abstract}
Magnetic resonance imaging (MRI) with diffusion-tensor imaging (DTI) together with a white matter fiber tracking (FT) technique was used to assess different brain white matter structures and functionalities in schizophrenic patients with typical first negative symptoms. In total, 30 schizophrenic patients with typical first negative symptoms, comprising an observation group were paired 1:1 according to gender, age, right-handedness, and education, with 30 healthy individuals in a control group. Individuals in each group underwent routine MRI and DTI examination of the brain, and diffusion-tensor tractography (DTT) data were obtained through whole brain analysis based on voxel and tractography. The results were expressed by fractional anisotropy (FA) values. The schizophrenic patients were evaluated using a positive and negative symptom scale (PANSS) as well as a Global Assessment Scale (GAS). The results of the study showed that routine MRIs identified no differences between the two groups. However, compared with the control group, the FA values obtained by DTT from the deep left prefrontal cortex, the right deep temporal lobe, the white matter of the inferior frontal gyrus and part of the corpus callosum were significantly lower in the observation group $(\mathrm{P}<0.05)$. The PANSS positive scale value in the observation group averaged $7.7 \pm 1.5$, and the negative scale averaged $46.6 \pm 5.9$, while the general psychopathology scale averaged $65.4 \pm 10.3$, and GAS averaged 53.8 \pm 19.2 . The Pearson statistical analysis, the left deep prefrontal cortex, the right deep temporal lobe, the white matter of the inferior frontal gyrus and the FA value of part of the corpus callosum in the observation group was negatively correlated with the negative scale $(\mathrm{P}<0.05)$, and positively correlated with GAS
\end{abstract}

Correspondence to: Dr Ying Zhang, Department of Geriatric, Tongde Hospital of Zhejiang Province, 234 Gucui Road, Hangzhou, Zhejiang 310012, P.R. China

E-mail: xstouykktn733@163.com

Key words: first negative symptoms, schizophrenia, magnetic resonance imaging diffusion-tensor imaging, white matter fiber tracking technique, fractional anisotropy, positive and negative symptom scale, Global Assessment Scale
$(\mathrm{P}<0.05)$. In conclusion, a decrease in the FA values of the left deep prefrontal cortex, the right deep temporal lobe, the white matter of the inferior frontal gyrus and part of the corpus callosum may be associated with schizophrenia with typical first negative symptoms and the application of MRI DTI-FT can improve diagnostic accuracy.

\section{Introduction}

Schizophrenia is a chronic disabling mental illness and has been listed as a major public health issue comparable to that of the HIV epidemic (1). The incidence of schizophrenia is $\sim 1 \%$ worldwide (2). Its cause, however, remains unclear. There have been advances in the treatment options to control symptoms but the prognosis for the majority of patients remains poor with the majority of patients staying incapacitated for life (3). New evidence seems to indicate that white matter abnormalities may be an important pathophysiological finding in schizophrenia (4); however, the results are inconsistent. Previous findings have shown that the seriousness of negative symptoms of schizophrenia may be associated with more intense white matter abnormalities (5), although other authors have found opposing results (6) complicating the analysis. The focus thus far has been on initial positive symptoms, therapeutic effects in patients with negative symptoms, social function and the extent of damage and prognosis, where there is a tendency to identify brain cell loss and degeneration (7).

Diffusion-tensor tractography (DTT) is a new magnetic resonance imaging (MRI) technology characterized by being non-invasive, not requiring exogenous contrast agents (8). It measures dispersion characteristics throughout nervous tissues, and is the only method providing high-quality biological tissue imaging to obtain clinical information, physical properties, microstructure and structural up-to-date data. Diffusion-tensor imaging (DTI) can identify abnormalities in the brain white matter microstructure, and is widely used in studies of brain connection structures in schizophrenia (9). Compared with single techniques, the combined applications of the region of interest (ROI), such as brain analysis based on voxel (VBA) and fiber tracking (FT) in DTI, as well as data statistical analysis methods have a higher diagnostic accuracy, and can produce real and reliable results (10). Different MRI analysis methods and FT have been combined and applied to children with retinal congenital malformations, congenital brain malformation, amyotrophic lateral sclerosis, computing 
capacity in developmental disorders as well as to healthy individuals, achieving promising results (11). However, to the best of our knowledge, this is the first study to be applied to schizophrenic patients.

A previous meta-analysis concluded that the white matter abnormalities of schizophrenia are mainly located on the left prefrontal deep and deep white matter in the left temporal lobe (12). However, findings of other studies have shown that schizophrenia with typical first negative symptoms is correlated with complete damage in the inferior frontal gyrus white matter (13). The present study used VBA and FT on structural integrity of white matter in schizophrenic patients with typical first negative symptoms to obtain more accurate results.

\section{Patients and methods}

Patient information. Patients diagnosed as schizophrenic at the Tongde Hospital of Zhejiang Province (Zhejiang, China) between June 2014 and December 2015 were continuously enrolled in the study. The diagnostic criteria conformed to those of the American Diagnostic and Statistical Manual of Mental Disorders, 4th edition (14), and two psychiatrists diagnosed each patient independently. Inclusion criteria for the study were: i) Negative scores in positive and negative symptom scale (PANSS) of $\geq 40$ as well as positive scores of $\leq 20$, ii) patients aged 16-60 years, iii) right-handedness and taking no psychotropic substances, and iv) guardians signed the informed consent. Exclusion criteria for the study were: i) MRI scan contraindications, ii) presence of severe organic disease, iii) history of psychoactive substance abuse, iv) history of traumatic brain injury or electric shock, v) pregnant and lactating women, and vi) symptoms of other psychiatric diseases confounding the diagnoses.

A total of 30 schizophrenic patients with typical first negative symptoms were selected for the observation group. Thirty right-handed healthy individuals served as the control group. The observation group included 12 men and 14 women, aged 23-58 years, average age of $39.7 \pm 11.6$ years, with the length of education time from 11 to 18 years, and an average of $13.5 \pm 4.4$ years, and disease history of 1-6 months. The control group included 13 men and 17 women, aged 21-56 years, average of $36.8 \pm 12.5$ years, with the length of education time from 12 to 19 years, and an average of $13.8 \pm 4.3$ years. Comparisons of gender, age and education time differences between the two groups yielded no statistically significant results $(\mathrm{P}>0.05)$. The ethics committee of the Tongde Hospital of Zhejiang Province approved this study.

Inspecting methods. A 3.0 T GE Signa TwinSpeed MRI machine (GE Healthcare, Piscataway, NJ, USA) was used to scan a patients education time of 12-19 years (with an average of 13 years). The patient was required to remain in a supine position wearing earplugs to reduce the noise from the machine (GEHealthcare, Piscataway, NJ, USA). The head was immobilized using foams as per the manufacturer's instructions.

The MRI data collection involved two separate steps: i) The image relevant to the clinical diagnosis was collected: A coronary spin echo sequence was used to obtain the T2-weighted and proton density-weighted images while the neuroimaging diagnosticians eliminated irrelevant data. ii) DTI data collection involved using the spin echo sequence to scan the plane parallel to the anterior and posterior joint line to obtain a diffusion-weighted imaging.

DTI data pre-treatment involved conversion of the original DICOM format images to analyze the format by DTI Studio (Pittsburgh, PA, USA). The b0 and fractional anisotropy (FA) parameters were also obtained. T-weighted images were normalized to the Montreal Neurological Institute (MNI) anatomical coordinates using the SPM5 (statistical parametric mapping) software implemented in the MATLAB 7.1 platform (MathWorks, Inc., Natick, MA, USA). Subsequently, the package was used to distribute each subject's b0 image to the T. A standard templte was constructed by MNI, and transformation parameters were applied to the FA images corresponding to each subject, thus standardizing the space of each subject's FA image. The 12-mm full width at the half maximum of Gaussian kernel was used to spatially smoothen each FA image after the standardization to increase the ratio of signal to noise, and have the data distribution align with the Gaussian distribution.

Indicators. The PANSS and Global Assessment Scale (GAS) were used to assess the clinical symptoms and determine the severity of the disease in each patient. At the same time, MRI and FA images were used to evaluate white matter functional connections between brain regions to identify any abnormalities, and determine whether there were correlations between the signs and clinical symptom types. PANSS included 7 positive scales (scores ranging from 7 to 49), 7 negative scales (scores ranging from 7 to 49 ), and 16 general psychopathology scales (scores ranging from 16 to 112). GAS scores ranged from 1 to 100 , and lower scores indicated severity of the disease.

Statistical analysis. SPSS 19.0 software (SPSS, Inc., Chicago, IL, USA) was used for the statistical analysis of the data obtained. Quantitative data were presented as mean \pm standard deviation, and the independent sample t-test was applied for comparison between groups. Qualitative data were expressed as cases and percentages, and the $\chi^{2}$ test was used for comparisons of groups. The Pearson correlation analysis was used to determine whether the data conformed to normal distribution. $\mathrm{P}<0.05$ was considered to indicate a statistically significant difference.

\section{Results}

Routine MRI findings. MRI images (Fig. 1) show no significant difference between the observation and control groups.

Comparison of FA values in different brain white matter regions on DTT. Compared with the control group, the FA values in the observation group shown by DTT were significantly lower in the left deep prefrontal cortex, the right deep temporal lobe, the white matter of the inferior frontal gyrus and part of the corpus callosum $(\mathrm{P}<0.05$; Fig. 2 and Table I).

Evaluation of PANSS and GAS. The average positive scale score of PANSS in the observation group was $7.7 \pm 1.5$, while the average negative scale score was $46.6 \pm 5.9$; with the general 
Table I. Comparison of FA values in different brain white matter sites on DTT.

\begin{tabular}{|c|c|c|c|c|c|c|}
\hline \multirow[b]{2}{*}{ Reduction area } & \multirow[b]{2}{*}{ Hemisphere } & \multicolumn{3}{|c|}{ MNI coordinate } & \multirow[b]{2}{*}{ Biggest difference in FA reduction value $(t)$} & \multirow[b]{2}{*}{ P-value } \\
\hline & & $\mathrm{X}$ & $\mathrm{Y}$ & $\mathrm{Z}$ & & \\
\hline \multirow[t]{2}{*}{ Deep prefrontal cortex } & Left & 13 & 29 & 33 & -4.632 & 0.036 \\
\hline & Right & -16 & 27 & 30 & 0.329 & 0.953 \\
\hline \multirow[t]{2}{*}{ Deep temporal lobe } & Left & -55 & -7 & -3 & 0.525 & 0.427 \\
\hline & Right & -46 & -5 & -2 & -4.957 & 0.034 \\
\hline Inferior gyrus frontalis & & 10 & 35 & 8 & -5.302 & 0.031 \\
\hline Part of corpus callosum & & -2 & 26 & 4 & -5.618 & 0.028 \\
\hline
\end{tabular}

FA, fractional anisotropy; DTT, diffusion-tensor tractography; MNI, Montreal Neurological Institute.
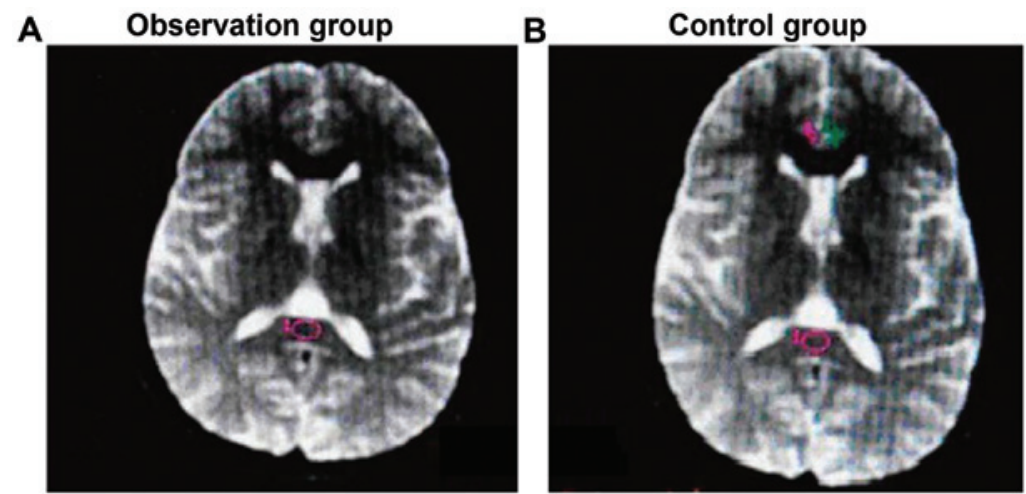

Figure 1. Routine brain magnetic resonance imaging (MRI) of the brain. (A) Routine T2WI MRI for the observation group, (B) routine T2WI MRI for the control group.
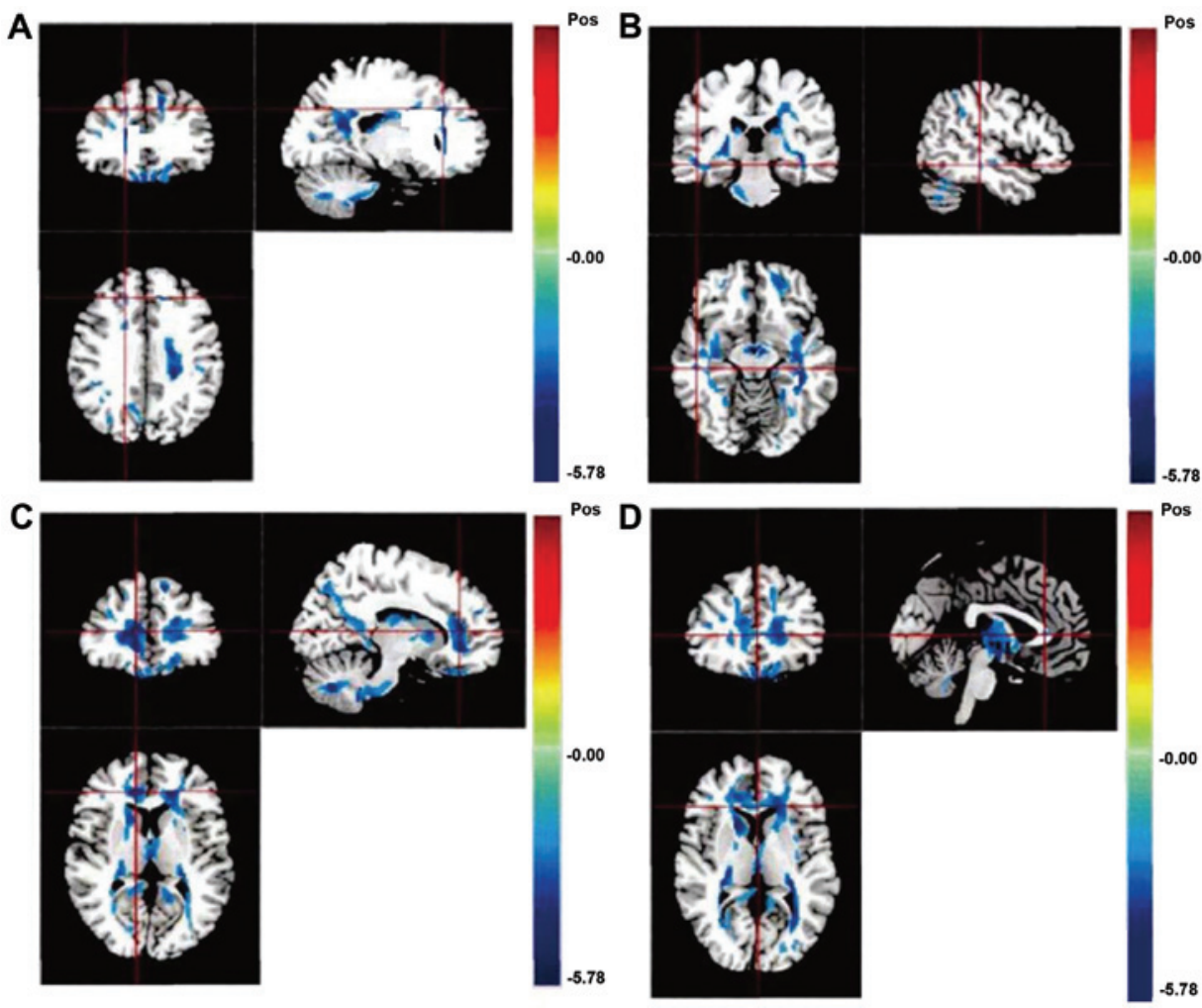

Figure 2. Diffusion-tensor tractography images for different brain sections in schizoprenic patients (lower fractional anisotropy values than those in the control brains). (A) Deep left prefrontal cortex, (B) deep right temporal lobe, (C) white matter of the inferior frontal gyrus, and (D) part of the corpus callosum. 
psychopathology scale average score being $65.4 \pm 10.3$. The GAS average score was $53.8 \pm 19.2$. According to the results of the Pearson correlation analysis, the FA values of the deep left prefrontal cortex, the deep right temporal lobe, the white matter of the inferior frontal gyrus and part of the corpus callosum had a negative correlation with the negative symptoms scales $(\mathrm{r}=0.432, \mathrm{P}=0.041 ; \mathrm{r}=0.475, \mathrm{P}=0.039 ; \mathrm{r}=0.512$, $\mathrm{P}=0.037 ; \mathrm{r}=0.533, \mathrm{P}=0.035)$, and a positive correlation with GAS ( $\mathrm{r}=0.468, \mathrm{P}=0.037 ; \mathrm{r}=0.477, \mathrm{P}=0.035 ; \mathrm{r}=0.496, \mathrm{P}=0.033$; $\mathrm{r}=0.503, \mathrm{P}=0.031)$.

\section{Discussion}

Positive and negative symptoms are independent of each other (15). A negative symptom is a reduction or loss of the normal function, consisting of a defective function, such as poor thought, bleak emotion, depression and passive social withdrawal. Negative symptoms can become stable over time and are associated with poor cognitive development, poor premorbid state, drug resistance and poor prognosis in the chronic phases (16). A type of schizophrenia with predominantly negative symptoms is classified as a special group of the disease. The analysis of the type of brain connections in cases presenting negative symptoms can reduce the heterogeneity effect of the sample on the study results, and can be useful in elucidating clearly the function of the abnormal brain connections in the pathogenesis of schizophrenia (16). Assuming that FA can reflect the nerve fiber connectivity of the white matter in the brain, the reduction of FA represents damage of regional cerebral white matter integrity and the reduction of nerve fiber connectivity. Previous findings have shown that the patients with schizophrenia mainly have structural abnormalities of the middle frontal lobe (complex of hippocampus-amygdala and entorhinal cortex), the superior frontal gyrus, the hippocampal sulcus, the corpus callosum, the frontal lobe and the cingulate gyrus (17).

DTI can demonstrate the shape of white matter tracts, allowing for observation of the structural characteristic of the brain's white matter, and revealing microchanges in the brain white matter nerve fiber tracts of schizophrenics, providing evidence for a possible neuropathological basis. FA is an important expression parameter of DTI, as it refers to the proportion of anisotropic water molecules in the whole diffusion tensor, and is able to reflect the diffusion anisotropy of water molecules and reveal the ordering of the tissue microstructures (18). Its size is correlated with the integrity of the myelin sheath, fiber compactness, and parallelism. Compared with relative anisotropic values and volume ratio values, FA values are characterized by less variability and higher imaging signal to noise ratio (19). DTT is the only non-invasive imaging method to display brain white matter fiber tracts on the living body; however, it lacks a golden standard to test its reliability. Tractography is divided into two methods: One involves line propagation techniques, and the other energy minimization techniques. The present study utilized the former technique, where seed and tracking conditions for the termination of the fiber bundle were established, and computers automatically displayed marked 3D fiber bundles, as previously described by Chung et al (20). The reliability of DTT results are influenced by image acquisition quality, applied algorithms, control parameters, ROI location and proficiency of relevant anatomical knowledge by the operator (21).

Among the brain structures identified as defective in the study, the corpus callosum is the largest commissural fiber that bears the function of information transfer and integration between the two cerebral hemispheres, including organizing hand movement and unifying emotion. Furthermore, it is associated with memory retrieval function, attention and wake-up states, and speech and hearing functions. It is an integral part of the active state of a person (22). It is generally considered that the left hemisphere of the brain is responsible for abstract thinking while the right one is responsible for imagery thinking (23). However, only by integrating abstract and imagery thinking constantly by means of connections can a person maintain a normal mode of thinking. When this connection is insufficient, abstract thinking and imagery thinking become fragmented, and thus abnormal thinking may occur (24).

In conclusion, the reduction of the FA values in the left deep prefrontal cortex, right deep temporal lobe, white matter of the inferior frontal gyrus and part of the corpus callosum may be associated with the pathogenesis of schizophrenia with typical first negative symptoms. Thus, the application of MRI DTI-FT is potentially valuable in improving diagnostic accuracy.

\section{Acknowledgements}

This study was supported by the Hospital-level issues, Zhejiang Tongde Hospital (Hangzhou, China), item no. 2012018.

\section{References}

1. No authors listed: Where next with psychiatric illness? Nature 336: 95-96, 1988.

2. Insel TR: Rethinking schizophrenia. Nature 468: 187-193, 2010.

3. Hegarty JD, Baldessarini RJ, Tohen M, Waternaux C and Oepen G: One hundred years of schizophrenia: A meta-analysis of the outcome literature. Am J Psychiatry 151: 1409-1416, 1994.

4. White T, Magnotta VA, Bockholt HJ, Williams S, Wallace S, Ehrlich S, Mueller BA, Ho BC, Jung RE, Clark VP, et al: Global white matter abnormalities in schizophrenia: A multisite diffusion tensor imaging study. Schizophr Bull 37: 222-232, 2011.

5. Miyata J, Sasamoto A, Koelkebeck K, Hirao K, Ueda K, Kawada R, Fujimoto S, Tanaka Y, Kubota M, Fukuyama H, et al: Abnormal asymmetry of white matter integrity in schizophrenia revealed by voxelwise diffusion tensor imaging. Hum Brain Mapp 33: 1741-1749, 2012.

6. Skelly LR, Calhoun V, Meda SA, Kim J, Mathalon DH and Pearlson GD: Diffusion tensor imaging in schizophrenia: Relationship to symptoms. Schizophr Res 98: 157-162, 2008.

7. Ayesa-Arriola R, Rodríguez-Sánchez JM, Suero ES, Reeves LE, Tabarés-Seisdedos R and Crespo-Facorro B: Diagnosis and neurocognitive profiles in first-episode non-affective psychosis patients. Eur Arch Psychiatry Clin Neurosci 1: 123-125, 2016.

8. Zhang XY, Fan FM, Chen DC, Tan YL, Tan SP, Hu K, Salas R, Kosten TR, Zunta-Soares G and Soares JC: Extensive white matter abnormalities and clinical symptoms in drug-naive patients with first-episode schizophrenia: a voxel-based diffusion tensor imaging study. J Clin Psychiatry 77: 205-211, 2016.

9. Mamata H, Mamata Y, Westin CF, Shenton ME, Kikinis R, Jolesz FA and Maier SE: High-resolution line scan diffusion tensor MR imaging of white matter fiber tract anatomy. AJNR Am J Neuroradiol 23: 67-75, 2002.

10. Pettersson-Yeo W, Allen P, Benetti S, McGuire P and Mechelli A: Dysconnectivity in schizophrenia: Where are we now? Neurosci Biobehav Rev 35: 1110-1124, 2011. 
11. Salmela MB, Cauley KA, Nickerson JP, Koski CJ and Filippi CG: Magnetic resonance diffusion tensor imaging (MRDTI) and tractography in children with septo-optic dysplasia. Pediatr Radiol 40: 708-713, 2010.

12. Ellison-Wright I and Bullmore E: Meta-analysis of diffusion tensor imaging studies in schizophrenia. Schizophr Res 108: 3-10, 2009.

13. Wolkin A, Choi SJ, Szilagyi S, Sanfilipo M, Rotrosen JP and Lim KO: Inferior frontal white matter anisotropy and negative symptoms of schizophrenia: A diffusion tensor imaging study. Am J Psychiatry 160: 572-574, 2003.

14. Rabe-Jablonska J: [Affective disorders in the fourth edition of the classification of mental disorders prepared by the American Psychiatric Association - diagnostic and statistical manual of mental disorders]. Psychiatr Pol 27: 269-279, 1993.

15. Strauss GP, Vertinski M, Vogel SJ, Ringdahl EN and Allen DN Negative symptoms in bipolar disorder and schizophrenia: A psychometric evaluation of the brief negative symptom scale across diagnostic categories. Schizophr Res 170: 285-289, 2016.

16. Siegrist K, Millier A,Amri I, Aballéa S and Toumi M: Association between social contact frequency and negative symptoms, psychosocial functioning and quality of life in patients with schizophrenia. Psychiatry Res 230: 860-866, 2015.

17. Wu CH, Hwang TJ, Chen YJ, Hsu YC, Lo YC, Liu CM, Hwu HG, Liu CC, Hsieh MH, Chien YL, et al: Primary and secondary alterations of white matter connectivity in schizophrenia: A study on first-episode and chronic patients using whole-brain tractography-based analysis. Schizophr Res 169: 54-61, 2015.
18. Meoded A, Faria AV, Hartman AL, Jallo GI, Mori S, Johnston MV, Huisman TA and Poretti A: Cerebral Reorganization after Hemispherectomy: A DTI Study. AJNR Am J Neuroradiol 14: $12-14,2016$

19. Lei W, Li N, Deng W, Li M, Huang C, Ma X, Wang Q, Guo W, Li Y, Jiang L, et al: White matter alterations in first episode treatment-naïve patients with deficit schizophrenia: A combined VBM and DTI study. Sci Rep 5: 12994, 2015

20. Chung MK, Adluru N, Lee JE, Lazar M, Lainhart JE and Alexander AL: Cosine series representation of 3D curves and its application to white matter fiber bundles in diffusion tensor imaging. Stat Interface 3: 69-80, 2010.

21. Chung MK, Adluru N, Lee JE, Lazar M, Lainhart JE and Alexander AL: Efficient parametric encoding scheme for white matter fiber bundles. Conf Proc IEEE Eng Med Biol Soc 2009: 6644-6647, 2009.

22. DelReEC,Konishi J,Bouix S,BloklandGA, Mesholam-GatelyRI, Goldstein J, Kubicki M, Wojcik J, Pasternak O, Seidman LJ, et al: Enlarged lateral ventricles inversely correlate with reduced corpus callosum central volume in first episode schizophrenia: Association with functional measures. Brain Imaging Behav 17: 156-158, 2015.

23. Whitehouse PJ: Imagery and verbal encoding in left and right hemisphere damaged patients. Brain Lang 14: 315-332, 1981.

24. Rigucci S, Santi G, Corigliano V, Imola A, Rossi-Espagnet C, Mancinelli I, De Pisa E, Manfredi G, Bozzao A, Carducci F, et al: White matter microstructure in ultra-high risk and first episode schizophrenia: A prospective study. Psychiatry Res 247: 42-48, 2016. 